\title{
COMPARAÇÃO DE MÉTODOS DE LABORATÓRIO E DE CAMPO PARA A ESTIMATIVA DA ÁREA FOLIAR EM FRUTEIRAS SILVESTRES
}

\author{
M.S. MIELKE ${ }^{1,2}$; A. HOFFMANN ${ }^{1}$; L. ENDRES ${ }^{1}$; J.C. FACHINELLO ${ }^{1}$

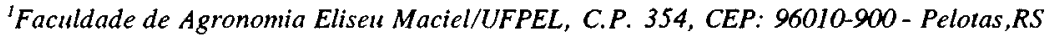 \\ ${ }^{2}$ Bolsista da FAPERGS
}

\begin{abstract}
RESUMO: Foi realizado um trabalho com o objetivo de estudar diferentes métodos de laboratório e de campo para a estimativa da área foliar de fruteiras silvestres, pertencentes à familia Myrtaceae. a saber: uvalheira (Eugenia uvalha Camb.), araçazeiro (Psidium cattleyanum Sabiue). goiabeira serraua (Feijoa sellowiana Berg.) e pitangueira (Eugenia uniflora DC). Entre os métodos de laboratório, também utilizados como "padrão", bons resultados foram obtidos utilizando-se um medidor automático de área foliar, planímetro ou através do método gravimétrico (fotocópia ou papel filtro). Dentre os métodos de campo, os melhores resultados foram obtidos por regressão linear. As estimativas das áreas foliares ( $y$ ) para as quatro especies estudadas podem ser tomadas a partir das equaçōes: $y=$ $2,658+0,554 X$, para o araçazeiro; $y=0.75 X$ ou $y=0,856 X-2,115$, para a goiabeira serrana; $y=0.68 X$, para a uvalheira $\mathrm{e} y=0,69 \mathrm{X}$ ou $\mathrm{y}=0,503+0,643 \mathrm{X}$, para a pitangueira, sendo $X=$ comprimento $\mathrm{x}$ largura da folha. Descritores: análise de crescimento, Eugenia uvalha, Psidium cattleyanum, Feijoa sellowiana, Eugenia uniflora
\end{abstract}

\section{COMPARISON OF LABORATORY AND FIELD METIIODS FOR TIIE ESTIMATION OF LEAF AREA OF WILD FRUIT SPECIES}

\begin{abstract}
This work was carried out in order to study different laboratory and field methods for estimating leaf area of wild fruit species, belonging to the Myrtaceae: "uvalha" plant (Eugenia uvalha Camb.), cattley guava plant (Psidium cattleyanum Sabine), feijoa plant (Feijoa sellowiana Berg) and Surinam cherry plant (Eugenia uniflora DC). Among the laboratory methods utilized as "standard methods", good results were obtained with the use of an automatic leaf area meter, planimeter or by the gravimetric method (photocopy or Whatman paper). Among field methods, best results were obtained with linear regression. The estimation of leaf area $y$ for all studied species could be obtained through the equations: $y=2,658+0,554 x$ for cattley guava plant; $y=0,75 x$ or $y=0,856 x-2,115$ for feijoa plant; $y=0,68 \times$ for "uvalha" plant and $y=0,69 \times$ or $y=0,503+0,643 \times$ for Surinam cherry plant. where $x=$ leaf length $x$ leaf width.
\end{abstract}

Key words: growth analisis, Eugenia uvalha, Psidium cattleyanum, Feijoa sellowiana, Eugenia uniflora

\section{INTRODUÇÃO}

Diversas espécies frutíferas silvestres do Rio Grande do Sul apresentam um bom potencial para exploração comercial e, neste sentido, a Faculdade de Agronomia Eliseu Macicl/FAEM/ UFPEL, vem realizando trabalhos com o objetivo de viabilizar o seu cultivo econômico. Estes trabalhos incluem a propagação vegctativa e a adaptação das plantas multiplicadas em diferentes ambientes (DUARTE, 1991; MIELKE, 1992; MIELKE et al., 1992). Dentre estas espécies, destacam-se a uvalheira, o araçazeiro, a gojabeira serrana e a pitangueira, devido ao seu potencial frutífero (para consumo "in natura" ou industrialização), ornamen- tal ou florestal (SANCHOTENE, 1989; MIELKE et al., 1990; RASEIRA \& RASEIRA, 1989).

O conhecimento de métodos para a determinação direta ou estimativa da área foliar é de grande importância em estudos que envolvem análise do crescimento de plantas, fotossíntese, propagação vegetativa, ataque de pragas e doenças, entre outros (REIS \& MÜLLER, 1979; LUCCHESI, 1984; BENINCASA, 1988).

São vários os métodos utilizados atualmente para a determinação da área foliar em plantas anuais ou perenes, os quais podem ser destrutivos ou de laboratório e não-destrutivos ou de campo. Segundo NORMAN \& CAMPBELL (1989), o uso de medidores automáticos e a medida 
a partir das dimensões da folha ou a partir das relações de peso da mesma constituem-se em métodos que podem ser utilizados para a determinação da área foliar.

Entre os métodos não-destrutivos estão aqueles que estimam a área foliar verdadeira através de medidas lineares tomadas nas folhas (comprimento $\mathrm{x}$ maior largura); através da equação de regressão linear entre as medidas lineares tomadas na folha e um método padrão, realizado em laboratório (BARROS et al., 1973; PINTO et al., 1979) ou através de um fator de correção (K), calculado através do quociente entre $O$ somatório das áreas calculadas pelo método padrão e o somatório das áreas calculadas pelas medidas lineares das folhas (BARROS et al. 1973).

Entre os métodos destrutivos ou de laboratório estão o método dos discos foliares, onde a área foliar real é estimada através de vazadores com área conhecida $e$ do peso do restante da folha; o método da pesagem das silhuetas, onde é feita a comparação entre o peso de uma área conhecida de papel com densidade definida e os pesos das silhuetas das folhas sobre os mesmos e o método da medição direta, realizada com uso de medidores automáticos de área foliar (automatic area meter) ou planímetro (HUERTA, 1962; PINTO et al., 1979; REIS \& MÜLLER, 1979; LUCCHESI, 1984; BENINCASA, 1988).

O presente trabalho teve por objetivo comparar diferentes métodos de laboratório e de campo para a estimativa da área foliar em 4 fruteiras silvestres do Rio Grande do Sul.

\section{MATERIAL E MÉTODOS}

Foram utilizadas folhas totalmente expandidas e com os bordos intactos, procedentes de plantas originadas de sementes, das seguintes espécies silvestres do Rio Grande do Sul: uvalheira (Eugenia uvalha Camb.), araçazeiro (Psidium cattleyanum Sabine), goiabeira serrana (Feijoa sellowiana Berg.) e pitangueira (Eugenia uniflora DC). As quatro espécies utilizadas são pertencentes à família Myrtaceae.

Foram adotados os seguintes métodos:

\section{Métodos "padrão" ou de laboratório}

Método gravimétrico - foram realizadas fotocópias das folhas sobre papel xerográfico comum (sulfite
$75 \mathrm{~g} / \mathrm{m}^{2}$ ). Também foram desenhados os perímetros das mesmas sobre papel filtro WHATMAN $n^{\circ} 42$. As silhuetas obtidas foram então pesadas com uso de uma balança analítica modelo Mettler-AE 166, e comparadas com áreas conhecidas dos mesmos papéis.

Método de pesagem dos discos foliares utilizando-se um vazadior com área conhecida, foram destacados discos foliares das porções basal, mediana e apical do limbo foliar. Através da área conhecida dos discos foliares destacados, do peso dos mesmos e do peso da folha, tomados através de uma balança analítica modelo Mettler-AE 166, foj estimada a área foliar total.

Medidor automático de área foliar - utilizando-se um medidor de área foliar modelo Li-Cor 3000 (Li-Cor Corporation, USA), foi realizada a leitura direta da área foliar.

Método do planímetro - através do uso de um planímetro, marca Koizumi - modelo KP- 27, foi determinada a área das fotocópias das folhas impressas sobre papel xerográfico (sulfite $\left.75 \mathrm{~g} / \mathrm{m}^{2}\right)$.

\section{Métodos estimadores ou de campo}

Método das figuras geométricas circunscritas - a partir das medidas do comprimento e maior largura das folhas, obtidas com paquímetro, foi calculada a área das elipses circunscrilas aos limbos foliares.

Modelo matematico - a estimativa da área foliar verdadeira foi calculada através da equação de regressão linear simples entre as áreas das figuras geométricas circunscritas (elipses) e as áreas calculadas pelo método padrâo ou através de um coeficiente de correção $(\mathrm{K})$, calculado pela seguinte equação:

$$
\mathrm{K}=\mathrm{E} \mathrm{AP} / \mathrm{E} \mathrm{AR} \text {; onde: }
$$

E AP - somatório das áreas calculadas pelo método padrão; e

E AR - somatório das áreas dos retângulos circunscritos aos limbos foliares.

Em função da disponibilidade de material, os métodos testados foram distribuídos entre as espécies estudadas da seguinte maneira: 


Espécies Métodos testados

Uvalheira 1.1 (Whatman 42, xerox), 1.2, 1.3, 1.4, 2.1 e 2.2

Araçazeiro 1.1 (Whatman 42, xerox), 1.2, 1.3, $1.4,2.1$ e 2.2

Goiabeira Serrana 1.1 (xerox), 1.2, 1.3, 1.4, 2.1 e 2.2 .

Pitangueira 1.1 (xerox), 1.3, 1.4, 2.1 e 2.2

Foi adotado o delineamento experimental em blocos ao acaso, com 10 plantas (blocos) e 20 folhas por planta. A comparação das médias foi realizada através do teste de Tukey.

\section{RESULTADOS E DISCUSSÃo}

As TABELAS 1, 2, 3 e 4 apresentam as médias obtidas para os diferentes métodos de laboratório e espécies estudadas. Entre as espécies, o araçazeiro apresentou a maior área foliar média, seguido da goiabeira serrana, da pitangueira e da uvalheira. Dentre os métodos, as áreas foliares médias mais elevadas foram obtidas através do método gravimétrico (fotocópia ou Whatman 42), enquanto que os valores mais baixos foram obtidos através dos discos foliares.

Segundo vários autores, para a determinação da área foliar através do método gravimétrico, a silhueta das mesmas pode ser desenhada em papel filtro com densidade homogênea (BENINCASA et al., 1976; LUCCHESI, 1984; BENINCASA, 1988) ou obtida através de fotocópia das mesmas em papel sulfite (HUERTA, 1962; BARROS et al., 1973; REIS \& MÜLLER, 1979; GOMIDE \& CASTRO NETO, 1989). Neste trabalho buscou-se comparar o uso de fotocópias e o desenho das silhuetas das folhas sobre papel Whatman 42. Na TABELA 5 são apresentadas as médias dos coeficientes de variação do peso de 50 discos com área constante de 5,27 $\mathrm{cm}^{2}$. Os discos de papel Whatman 42 apresentaram peso e uniformidade superiores ao papel sulfite, mas não foram encontradas diferenças significativas entre as médias calculadas por estes dois métodos, nas espécies em que foram testados (TABELAS 1 e 2). Devido ao alto custo do papel Whatman, ou outro de mesma qualidade, e também a possíveis erros que ocorreram, presumivelmente devido à superestimativa da área foliar por variaçōes ocorridas no corte da silhueta, além da existência de ampla bibliografia na qual é utilizado o método das fotocópias como padrão (GOMIDE et al., 1977; PINTO et al., 1979; ABRAHÃO, 1981; GOMIDE, 1989), verifica-se que, dependendo do grau de precisão exigido no experimento, $o$ uso de fotocópias para a determinação da área foliar através do método gravimétrico é viável. Através deste método podem ser obtidos resultados relativamente seguros $e$ de forma bastante econômica.

TABELA 1. Médias $\left(\mathrm{cm}^{2}\right)$ e desvio padrão dos diferentes métodos de laboratório testados para a determinação da área foliar de uvalheira.

\begin{tabular}{lc}
\hline Método & Média $+/$ - desvio padrão \\
\hline Whatman 42 & $6,53+/-0,37 \mathrm{a}$ \\
Fotocópia & $6,40+/-0,39 \mathrm{a}$ \\
Planímetro & $6,04+/-0,36 \mathrm{~b}$ \\
Medidor automático de & \\
área foliar & $5,97+/-0,37 \mathrm{~b}$ \\
Discos foliares apicais & $3,65+/-0,27 \mathrm{c}$ \\
Discos foliares medianos & $3,51+/-0,29 \mathrm{c}$ \\
Discos foliares basais & $3,50+/-0,33 \mathrm{c}$ \\
\hline
\end{tabular}

D.M.S. Tukey $5 \%=0,34$

Média geral $=4,87 \mathrm{~cm}^{2}$

C.V. $=4,99 \%$

O método dos discos foliares subestimou as áreas foliares nas espécies em que foi testado. Somente para a goiabeira serrana as áreas calculadas através de discos retirados da porção apical das folhas não diferiram significativamente das áreas calculadas através do medidor automático de área foliar, do método gravimétrico e do planímetro (TABELA 3). Assim, os resultados apresentados são inversos aos de GOMIDE et al. (1977) e PINTO et al. (1979), onde os discos foliares retirados das porções basais, medianas e apicais, mesmo não diferindo significativamente do método padrão utilizado (fotocópia), superestimaram a área foliar em cafeeiro (Coffea arabica L.) e goiabeira (Psidium guajava L.), respectivamente. Da mesma forma, estes resultados foram inversos à afirmação de Huck \& Bolas (1956), citado por 
HUERTA (1962), de que existe uma variação decrescente do peso da lâmina e da nervura central desde a base até o ápice. Assim, pode-se observar que este método é variável de acordo com a espécie utilizada e deve sempre ser comparado a outros métodos, levando-se em conta as características morfológicas da espécie em estudo. Ross (1981), citado por NORMAN \& CAMPBELL (1989), recomenda que o disco foliar seja retirado de uma porção da folha onde a relação peso da matéria seca/área seja igual à média de toda a folha.

TABElA 2. Média $\left(\mathrm{cm}^{2}\right)$ e desvio padrão dos diferentes métodos de laboratório testados para a determinação da área foliar de araçazeiro.

\begin{tabular}{|c|c|}
\hline Método & +1- desvio padrão \\
\hline Fotocópia & $17,32+/-2,68 \mathrm{a}$ \\
\hline Whatman 42 & $17,27+/-2,65 a b$ \\
\hline Planímetro & $16,86+1-2,66 a b$ \\
\hline $\begin{array}{l}\text { Medidor automático } \\
\text { de área foliar }\end{array}$ & $16,51+/-2,57$ bc \\
\hline Discos foliares apicais & $15,87+/-2,44 \quad c$ \\
\hline Discos foliares medianos & $14,78+/-1,98$ \\
\hline Discos foliares basais & $13,65+/-1,61$ \\
\hline
\end{tabular}

D.M.S. Tukey $5 \%=0,92$

Média geral: $15,87 \mathrm{~cm}^{2}$

C.V. $=3,53 \%$

Pela revisão da literatura, observou-se que a maioria dos trabalhos realizados utilizou o método gravimétrico ou a medição direta através de planímetro como padråo (HUERTA, 1962; BARROS et al., 1973; KARIKARI, 1973; BENINCASA et al., 1976; GOMIDE et al., 1977; PINTO et al., 1979; ABRAHÃO, 1981; GOMIDE \& CASTRO NETO, 1989). Por outro lado, a utilização de medidores automáticos de área foliar proporciona um grau de aferimento e facilidade de operação muito superiores aos demais métodos, tendo como único problema o alto custo do equipamento (PINTO et al., 1979; BENINCASA, 1988; GOMIDE \& CASTRO NETO, 1989, NORMAN \& CAMPBELL, 1989). Conforme pode-se observar nas TABELAS 1, 2, 3 e 4, as áreas medidas determinadas através da medição direta, por planímetro ou medidor automático de área foliar, não diferiram significativamente entre si em nenhuma das 4 espécies. Dessa forma, pode-se aconselhar o uso preferencial destes dois métodos para a utilização como padrão. Por outro lado, dependendo das condições financeiras existentes, assim como do local onde, será desenvolvido o trabalho e do grau de precisão exigido, o uso do método gravimétrico, através de papel filtro ou fotocópia, pode ser aconselhável.

TABELA 3. Médias $\left(\mathrm{cm}^{2}\right)$ e desvio padrão dos diferentes métodos de laboratório testados para a determinação da área foliar de goiabeira serrana.

Método Média $+/$-desvio padrão

$\begin{array}{ll}\text { Medidor automático } & \\ \text { de área foliar } & 15,68+/-2,48 \mathrm{a} \\ \text { Fotocópia } & 15,60+/-2,41 \mathrm{a} \\ \text { Discos foliares apicais } & 15,53+/-2,41 \mathrm{a} \\ \text { Planimetro } & 15,27+/-2,33 \mathrm{a} \\ \text { Discos foliares medianos } & 14,30+/-1,93 \mathrm{~b} \\ \text { Discos foliares basais } & 12,99+1-1,58 \mathrm{c}\end{array}$

D.M.S. Tukey $5 \%=0,87$

Média geral $=14,79 \mathrm{~cm}^{2}$

C.V. $=4,31 \%$

TABElA 4. Médias $\left(\mathrm{cm}^{2}\right)$ e desvio padrão dos diferentes métodos de laboratório testados para a determinação da área foliar de pitangueira.

Método

Média $+/$ - desvio padrão

Medidor automático de

\begin{tabular}{ll} 
área foliar & $7,85+/-1,15$ a \\
Fotocópia & $7,85+/-1,18 \mathrm{a}$ \\
Planímetro & $7,83+/-1,13 \mathrm{a}$ \\
\hline
\end{tabular}

D.M.S. Tukey $5 \%=0,13$

Média geral: $7,84 \mathrm{~cm}^{2}$

C.V. $=1,43 \%$

Em face aos resultados discutidos acima, foram realizadas regressões lineares entre as áreas obtidas pelo medidor automático de área foliar, planímetro, papel sulfite (fotocópia) e papel filtro (Whatman 42) e as medidas de comprimento e maior largura das folhas nas quatro especies estudadas (TABELA 6). Observou-se que houve um comportamento diferenciado entre os métodos, dentro das espécies, para os coeficientes de determinação $\left(r^{2}\right)$ encontrados. Assim, foram selecionados os 
métodos que não diferiram significativamente do medidor automático de área foliar ou do planímetro e onde obteve-se os maiores valores de $r^{2}$ (medidor automático de área foliar para a uvalheira, planímetro para o araçazeiro e goiabeira serrana e fotocópia para a pitangueira), os quais foram utilizados como padrão para a determinação da área foliar através dos métodos de campo.

TABELA 5. Médias e desvio padrão do peso (g) de 50 discos recortados de papel sulfite (fotocópia) e Whatman 42 com $5,27 \mathrm{~cm}^{2}$ cada.

\begin{tabular}{lr}
\hline Método & Média $+/$ - desvio padrão \\
\hline Sulfite $\left(75 \mathrm{~g} / \mathrm{m}^{2}\right)$ & $0,0408+/-0,0012$ \\
Whatman 42 & $0,0544+/-0,0009$ \\
\hline
\end{tabular}

Observando mais atentamente a TABELA 6, nota-se um $r^{2}$ muito baixo para a regressão linear entre planímetro $\mathrm{x}$ área do retângulo $(\mathrm{L} \times \mathrm{l}$ ) para folhas de uvalheira, ao passo que a regressão entre o medidor automático de área foliar $x$ área do retângulo $(L \times 1)$ apresentou um $r^{2}$ bastante elevado. Este fato demonstra a baixa precisão do planímetro quando utilizado para a determinação da área de folhas pequenas, visto que, entre as espécies testadas, a menor área foliar média foi encontrada nesta espécie $\left(<6,5 \mathrm{~cm}^{2}\right.$ ). Outro dado interessante observado na TABELA 6 foram os altos valores de $r^{2}$ encontrados para as regressões entre o método gravimétrico através de fotocópia $\mathrm{e}$ as áreas dos retângulos nas quatro espécies estudadas. Embora deva ser feita uma observação no sentido de que as medidas de comprimento e maior largura foram tomadas sobre as fotocópias e não sobre as folhas "in vivo", estes dados vêm a fortalecer as discussões anteriores sobre a possível utilização do método das fotocópias como padrão.

Nas TABELAS $6,7,8$ e 9 são apresentadas as médias obtidas através dos métodos estimadores ou de campo (elipse circunscrita, coeficiente $\mathbf{K}$ e regressão), comparados com o respectivo padrão. Nota-se que a equação da elipse superestimou a área foliar para as quatro espécies estudadas, enquanto que a equação da regressão subestimou a área foliar para a uvalheira.

De maneira geral, os melhores resultados foram obtidos através do uso de regressão linear ou do coeficiente K. Assim, as estimativas das áreas foliares para as quatro espécies estudadas podem ser tomadas a partir das equações:

$y=0,68 X$, para a uvalheira;

$y=0,66 X$ ou $y=2,658+0,554 X$, para $o$ araçazeiro;

$y=0,75 X$ ou $y=0,856 X-2,115$, para a goiabeira serrana;

$y=0,69 X$ ou $y=0,503+0,643 X$, para $a$ pitangueira;

onde $\mathrm{X}=$ comprimento $(\mathrm{L}) \times$ largura $(\mathrm{I}) \mathrm{da}$ folha.

TABELA 6. Equações de regressão linear simples e coeficientes de determinação entre métodos de laboratório $\mathrm{x}$ área dos retângulos tomados à partir do comprimento (L) e maior largura (I).

\begin{tabular}{|c|c|c|c|c|}
\hline \multirow{2}{*}{ Espécie } & \multicolumn{4}{|c|}{ Método } \\
\hline & Planímetro & $\begin{array}{l}\text { Medidor automático } \\
\text { de área foliar }\end{array}$ & Whatman 42 & Fotocópia \\
\hline Uvalheira & $\begin{array}{l}y=7,871-0,202 x \\
r^{2}=0,101 \mathrm{~ns}\end{array}$ & $\begin{array}{l}y=0,053+0,669 X \\
r^{2}=0,978 * *\end{array}$ & $\begin{array}{l}y=0,750+0,654 X \\
r^{2}=0,935 * *\end{array}$ & $\begin{array}{l}y=0,254+0,696 x \\
r^{2}=0,985 * *\end{array}$ \\
\hline Araçazeiro & $\begin{array}{l}y=2,658+0,554 X \\
r^{2}=0,660 n s\end{array}$ & $\begin{array}{l}y=3,846+0,493 x \\
r^{2}=0,563 * *\end{array}$ & $\begin{array}{l}y=3,575+0,534 X \\
r^{2}=0,617 * *\end{array}$ & $\begin{array}{l}y=0,884 X-2,362 \\
r^{2}=0,986 * *\end{array}$ \\
\hline $\begin{array}{l}\text { Goiabeira } \\
\text { serrana }\end{array}$ & $\begin{array}{l}y=0,856 x-2,115 \\
r^{2}=0,988 * *\end{array}$ & $\begin{array}{l}y=0,833 x-1,247 \\
r^{2}=0,824 * *\end{array}$ & -- & $\begin{array}{l}y=0,884 X-2,362 \\
r^{2}=0,986 * *\end{array}$ \\
\hline Pitangueira & $\begin{array}{l}y=0,869+0,609 x \\
r^{2}=0,947 * *\end{array}$ & $\begin{array}{l}y=0,709+0,625 X \\
r^{2}=0,973 * *\end{array}$ & - & $\begin{array}{l}y=0,503+0,643 x \\
r^{2}=0,983 * *\end{array}$ \\
\hline
\end{tabular}

$\mathrm{X}$ = área do retângulo $(\mathrm{L} \times \mathrm{l})$ 
TABELA 7. Médias $\left(\mathrm{cm}^{2}\right)$ e desvios padrão dos diferentes métodos de laboratório testados para a determinação da área foliar de uvalheira.

\begin{tabular}{lr}
\hline Método & Média +/-desvio padrão \\
\hline Elipse & $6,94+/-0,43 \mathrm{a}$ \\
$\begin{array}{l}\text { Coeficiente } \mathrm{K}(\mathrm{y}=0,68 \mathrm{X}) \\
\text { Medidor automático de }\end{array}$ & $6,01+/-0,38 \mathrm{~b}$ \\
$\begin{array}{l}\text { área foliar } \\
\text { Regressão }(\mathrm{y}=0,053+\end{array}$ & $5,97+/-0,37 \mathrm{~b}$ \\
$+0,663 \mathrm{x})$ & $5,91+/-0,37 \mathrm{c}$ \\
\hline
\end{tabular}

D.M.S. Tukey $5 \%=0,05$

Média geral $=6,21 \mathrm{~cm}^{2}$

C.V. $=0,68 \%$

TABELA 8. Médias $\left(\mathrm{cm}^{2}\right)$ e desvios padrão dos diferentes métodos de laboratório testados para a determinação da área foliar de araçazeiro.

\begin{tabular}{ll}
\hline Método & Média $+/$ - desvio padrão \\
\hline Elipse & $20,15+/-3,07 \mathrm{a}$ \\
Coeficiente $\mathrm{K}(\mathrm{y}=0,66 \mathrm{X})$ & $16,93+/-2,58 \mathrm{~b}$ \\
Planimetro & $16,86+/-2,66 \mathrm{~b}$ \\
Regressão $(\mathrm{y}=2,658+$ & \\
$+0,554 \mathrm{x})$ & $16,83+/-2,16 \mathrm{~b}$ \\
\hline
\end{tabular}

D.M.S. Tukey $5 \%=1,09$

Média geral $=17,69 \mathrm{~cm}^{2}$

C.V. $=5,04 \%$

TABELA 9. Médias $\left(\mathrm{cm}^{2}\right)$ e desvios padrão dos diferentes métodos de laboratório testados para a determinação da área foliar de goiabeira serrana.

\begin{tabular}{ll}
\hline Método & Média $+/$-desvio padrão \\
\hline Elipse & $15,96+/-2,13 \mathrm{a}$ \\
Coeficiente $\mathrm{K}(\mathrm{y}=0,75 \mathrm{X})$ & $15,34+/-2,03 \mathrm{~b}$ \\
Regressão $(\mathrm{y}=0,856 \mathrm{x}-$ & \\
$2,115)$ & $15,28+/-2,32 \mathrm{~b}$ \\
Planímetro & $15,27+/-2,33 \mathrm{~b}$ \\
\hline
\end{tabular}

D.M.S. Tukey $5 \%=0,31$

Média geral $=15,46 \mathrm{~cm}^{2}$

C.V. $=1,65 \%$

Sci. agric., Piracicaba, 52(1):82-88, jan./abr. 1995
TABELA 10. Médias $\left(\mathrm{cm}^{2}\right)$ e desvios padrão dos diferentes métodos de laboratório testados para a determinação da área foliar de pitangueira.

\begin{tabular}{ll}
\hline Método & Média $+/$ - desvio padrão \\
\hline Elipse & $8,79+/-1,80 \mathrm{a}$ \\
Coeficiente $\mathrm{K}(\mathrm{y}=0,69 \mathrm{X})$ & $7,89+/-1,25 \mathrm{~b}$ \\
Regressão $(\mathrm{y}=0,503+$ & \\
$+0,643 \mathrm{x})$ & $7,85+/-1,16 \mathrm{~b}$ \\
Planímetro & $7,85+/-1,18 \mathrm{~b}$ \\
\hline
\end{tabular}

D.M.S. Tukey $5 \%=0,46$

Média geral $=8,10 \mathrm{~cm}^{2}$

C.V. $=4,68 \%$

Com base nos resultados acima apresentados e discutidos, nota-se que existe uma certa variação de acordo com o método ou espécie estudada. Assim, a escolha do método de laboratório, utilizado como padrão, ou o método de campo a ser adotado, deve sempre levar em conta fatores como o grau de precisão exigido no experimento, as características morfológicas e fisiológicas da espécie estudada e os recursos financeiros disponíveis.

\section{REFERÊNCIAS BIBLIOGRÁFICAS}

ABRAHÃO, E.; CHALFUN, N.N.J. Comparação entre métodos de determinação da área foliar em videira "Seyve Villard 12.375". Ciência e Prática. Lavras, v.5, n.1, p.55-58, 1981 .

BARROS, R.S.; MAESTRI, M.; VIEIRA, J.; BRAGA FILHO, L.J. Determinação da área foliar em café (Coffea arabica L. cv. 'Bourbon Amarelo'). Revista Ceres, Viçosa, v.20, n.107, p.44-52, 1973.

BENINCASA, M.M.P.; BENINCASA, M.; LATANZE, R.J.; JUNQUETTI, M.T.G. Método não-destrutivo para estimativa da área foliar de Phaseolus vulgaris $\mathrm{L}$. (feijoeiro). Científica, Marilia, v.4, n.1, p.43-48, 1976.

BENINCASA, M.M.P. Analise do crescimento de plantas. Jaboticabal: FUNEP, 1988. 42p.

DUBOIS, L.A.M. Variation for the adventitious root formation of dwarf rose cuttings with regard to the leaf area. In: INTERNATIONAL HORTICULTURAL CONGRESS, 23, Firenze, 1990. Abstracts of Contribuited Papers... p.193. 
DUARTE, O.R. Multiplicação da goiabeira serrana (Feijoa sellowiana Berg) através de estacas semilenhosas. Pelotas, 1991. 82p. Dissertação (Mestrado) - Faculdade de Agronomia Eliseu Maciel, Universidade Federal de Pelotas.

GOMIDE, M.B.; LEMOS, O.V.; TOURINO, D.; CARVALHO, M.M.; CARVALHO, J.G.; DUARTE, C.S. Comparação entre métodos de determinação de área foliar em cafeeiros Mundo Novo e Catuí. Ciência e Prática, Lavras, v.1, n.2, p.118-123, 1977.

GOMIDE, M.B.; CASTRO NETO. P. Determinação da área foliar "in vitro" no feijoeiro (Phaseolus vulgaris L.). I - metodologia para a cultivar Eriparza. Ciência e Prática. Lavras, v.13, n.2. p.152-155, 1989.

HUERTA. A.S. Comparación de métodos de laboratório y de campo para el area del cafeto. Cenicafé. Chinchiná, v.13, n.1, p.33-42. 1962.

KARIKARI. S.K. Estimation of leaf area in papaya (Carica papaya) from leaf measurements. Tropical Agriculture. Trinidad, v.50, n.4. 1973.

LUCCHESI. A.A. Utilização prática da análise de crescimento vegetal. Anais da Escola Superior de Agricultura "Luiz de Queiroz". Piracicaba, v.41. p.181-202. 1984

MIELKE. M.S. Multiplicação de goiabeira serrana (Feijoa sellowiana Berg) através de enxertia. Pelotas. 1992. 46p. Dissertação (Mestrado) Faculdade de Agronomia Eliseu Maciel. Universidade Federal de Pelotas.

MIELKE, M.S.; FACHINELLO, J.C.: RASEIRA, A. Fruteiras nativas - características de 5 Mirtáceas com potencial para utilizaçāo comercial. Hortisul, Pelotas, v. 1, n.2, p.32-36, 1990.
MIELKE, M.S.; SANTOS, B.G.F.; SANTOS, D.S.B.; FACHINELLO, J.C. Balanço hídrico de plantas de goiabeira serrana (Feijoa sellowiana Berg) submetidas a déficit hidrico. In: CONGRESSO IBEROAMERICANO DE HORTICULTURA, 1, Montevideo, 1992. Resumos. Montevideo: Sociedad Uruguaya de Horticultura, 1992. p.8.

NORMAN, J.M.; CAMPBELL, G.S. Canopy structure. In: PEARCY, R.W.; EHLERINGER, J.R.; MOONEY, H.A.: RUNDEL. P.W. Plant physiological ecology - field methods and instrumentation. London: Chapman and Hall. 1989. p. $301-325$.

PINTO, A.C.Q.; HOSTALACIO. S.; GOMIDE. M.B.; OLIVEIRA. L.E.M. Comparação de mútodos de determinaçâo da áréa foliar na cultura da goiahcira (Psidium guajava L.). Ciência e Prática, Lavras, v.3, n.1.p.58-62. 1979.

RASEIRA, A.: RASEIRA, M.C.B. Fruteiras nativas de clima temperado. Hortisul, Pelotas, v.1, n.2. p.47-.51, 1989.

REIS, G.G. dos: MÜller, M.W. Análise de crescimento de plantas - mensuração do crescimento Belém: FCAP. 1979.39p. (FCAP - Informe Didático, 1)

SANCHOTENE. M.C.C. Fruteiras mativas úteis à fauna na arborização urbana. Porto Alegre: FEPLAM, 1989. $311 \mathrm{p}$.

Entregue para publicação em 03.05 .94

Aceito para publicação em 01.08 .94 